\title{
Des chercheurs en herbe à l'école Boucicaut
}

\author{
Évelyne Fillon $^{(1)}$, Maud Lebreton ${ }^{(2)}$, Béatrice Madignier ${ }^{(2)}$, Valérie Polge ${ }^{(3)}$ \\ et Yves Noat ${ }^{(4)}$ (yves.noat@insp.jussieu.fr) \\ (1) Ancienne directrice de l'école élémentaire Boucicaut \\ (actuellement directrice de l'école primaire Littré, 6 rue Littré, 75006 Paris). \\ (2) Professeures des écoles à l'école élémentaire Boucicaut, 78 rue de la Convention, 75015 Paris. \\ (3) Ancienne professeure des écoles à l'école élémentaire Boucicaut \\ (actuellement à l'école élémentaire 188 rue d’Alésia, 75014 Paris). \\ (4) Chercheur au CNRS, Institut des Nanosciences de Paris, 4 place Jussieu, 75005 Paris.
}

Ce texte est le témoignage

d'une expérience pédagogique

menée à l'école élémentaire

Boucicaut, située dans

le $15^{\mathrm{e}}$ arrondissement de Paris, dans les murs de l'ancien

hôpital Boucicaut.

Pendant plusieurs années,

de 2004 à 2010, sous l'impulsion

d'un petit groupe d'enseignants

et d'un physicien du CNRS,

de jeunes élèves du CP au CM1

sont devenus de véritables

chercheurs en herbe et ont

expérimenté un apprentissage

de la science créatif et non

scolaire.

Ces ateliers s'inscrivent dans

la lignée d'autres initiatives

de ce type, telle que celle de

I'association « La main à la

pâte », lancée par le prix Nobel

de physique 1996 G. Charpak.
L'expérience décrite dans cet article est d'abord née d'un hasard : la proximité géographique d'un laboratoire de recherche et d'une école. En effet, tout a débuté à la suite du déménagement - pour cause de désamiantage - du laboratoire de recherche en physique de la matière condensée, du grand campus de Jussieu situé dans le Quartier latin, vers le $15^{\mathrm{e}}$ arrondissement de Paris, dans l'ancien hôpital Boucicaut.

Un jour, un chercheur du CNRS est venu proposer ses services à la directrice de l'école, pour enseigner les sciences dans une de ses classes. Ainsi ont débuté les ateliers sciences à l'école élémentaire Boucicaut, qui ont fonctionné - avec trois professeures des écoles différentes - pendant six années scolaires consécutives, de septembre 2004 à juin 2010.

La science expérimentale a-t-elle sa place au sein de l'école ? Quel rôle peut-elle y jouer? Voici les questions que nous nous sommes posées au terme de ces années d'ateliers et auxquelles nous tentons ici de donner des éléments de réponse.

\section{L'esprit de la démarche}

«La joie de contempler et de comprendre, voilà le langage que me porte la nature " nous dit Einstein [1]. Le scientifique est dans une perpétuelle quête d'une meilleure compréhension de la nature. En ce sens, la science s'apparente à l'art et son apprentissage, comme celui d'un instrument de musique ou de la peinture, nécessite une pratique assidue sur une longue durée. Ainsi, c'est au travers d'un travail de fond que le goût pour la science a la plus grande chance de naître et de se développer.

Pour cette raison, plutôt que d'intervenir ponctuellement dans des classes, nous avons décidé de suivre une seule et même classe durant une ou deux années scolaires consécutives, et de mettre en place des ateliers dans un esprit proche de celui de la recherche, telle qu'on la trouve dans les laboratoires de physique.

\section{Le cadre des ateliers}

Au début, nous avions prévu de faire une séance tous les quinze jours. Mais en pratique, un rythme d'une fois par semaine - quand cela était possible - s'est révélé beaucoup plus productif. En effet, nous nous sommes vite rendus compte que des séances trop espacées nuisaient à la continuité de la pensée, nécessaire dans cette démarche.

La première année, grâce à la présence de locaux inoccupés, nous disposions d'une salle où nous pouvions laisser en place nos expériences en cours. Cette situation était idéale pour des raisons pratiques évidentes, mais aussi parce que le changement de lieu facilite à bien des égards l'accès à un autre espace, celui de la création.

Il a été très vite clair que nous ne souhaitions pas intervenir en tant que "professeur", souvent perçu par les élèves comme « celui qui sait ", mais plutôt en tant que « chercheur 


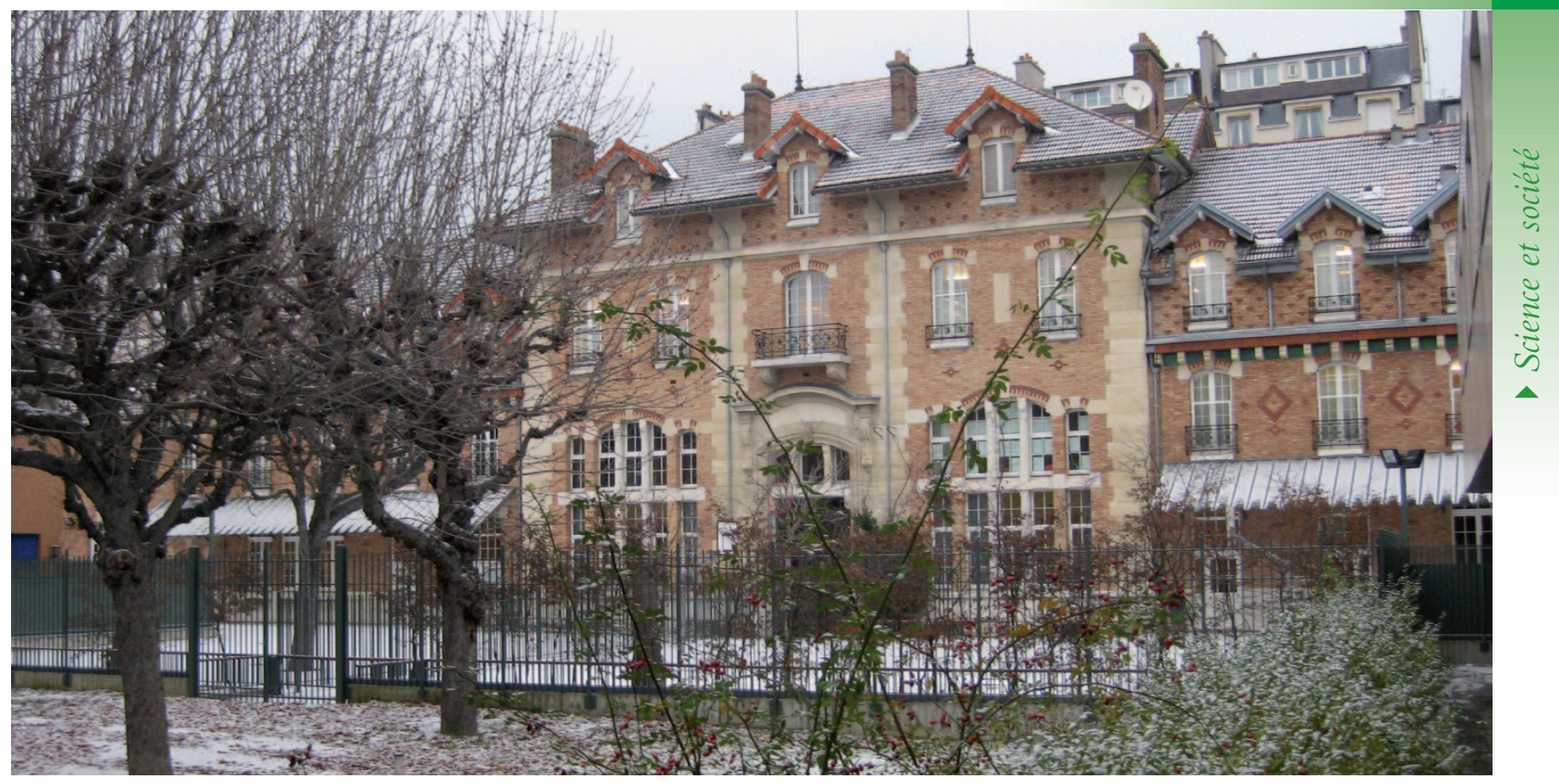

L'école élémentaire Boucicaut en décembre 2010.

de vérité ", pour faire partager notre curiosité et favoriser une démarche active et la plus autonome possible vers la connaissance. Quelle fut l'idée fondatrice de notre approche ? Elle était fort simple : amener les enfants à se questionner sur un thème choisi, puis les accompagner dans une démarche réflexive et expérimentale pour tenter de trouver et d'élaborer des réponses... En raison de la valeur que nous accordons au questionnement et à la recherche de réponse par soi-même, nous nous sommes obligés à ne pas assener des réponses "toutes faites " et à laisser la

(1)
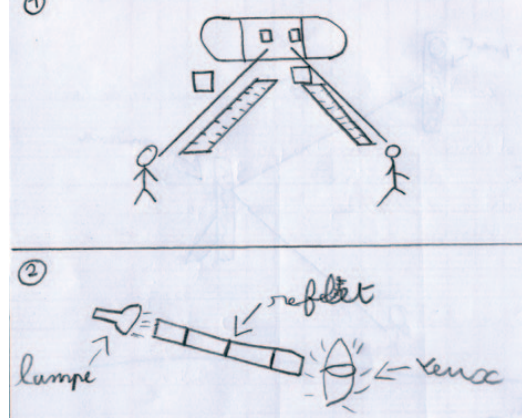

$$
\text { (3) } \begin{aligned}
& \text { Chiffon } \\
& \ll \text { consières }
\end{aligned}
$$

1. Schéma extrait du cahier d'expériences d'un élève, lors d'une séance sur la lumière. plupart du temps les enfants dans leurs interrogations, et parfois dans la frustration qui en découle !

À l'image du chercheur qui possède son fameux " cahier de manip " où il consigne soigneusement les paramètres de chaque expérience, chaque enfant a aussi un " cahier d'expériences " sur lequel il en dessine les schémas (fig. 1) et présente les conclusions tirées des mises en commun. Un travail écrit est fait régulièrement.

À partir du questionnement, les élèves proposent des expériences et nous les guidons dans cette démarche. Nous n'avions pour travailler que peu de matériel disponible. C'était surtout de la "débrouille » et de l'improvisation : récupération d'objets glanés ici ou là, achats de dernière minute au supermarché du coin...

Une séance se divisait donc en plusieurs temps :

- la mise en place du questionnement ;

- le travail expérimental, qui se faisait généralement par petits groupes de 2 à 4 élèves ;

- une mise en commun et une discussion avec toute la classe ;

- le passage à l'écrit, la schématisation de l'expérience et les conclusions issues de la mise en commun.

Ces conclusions étaient parfois reformulées par la maîtresse, qui précisait aussi le vocabulaire faisant défaut pour désigner un phénomène identifié par les élèves (la vaporisation, l'ébullition...).

\section{Les différents thèmes} et la façon de les aborder

De nombreux thèmes ont été abordés dans les différents niveaux : les états de l'eau, "flotte et coule ", la " peau de l'eau » (i.e. la tension de surface), l'air, la lumière, l'électricité, le son, l'énergie, le temps et l'espace, la gravitation...

La façon d'aborder un thème n'était pas unique, loin de là. Nous recherchions à chaque fois un angle d'approche nouveau, le but étant de susciter l'étonnement et un questionnement : un défi posé à la classe (Peut-on faire flotter un trombone ?), une réflexion autour d'une notion ou d'un concept, une expérience, ou encore un simple objet (un instrument de musique) en guise de point de départ.

Nous démarrions souvent par un défi : Comment faire flotter un bout de pâte à modeler (thème "flotte-coule ") ou encore un trombone sur l'eau (pour aborder la notion de tension de surface) ? Certains thèmes ont été introduits par une réflexion commune à bâtons rompus autour d'une notion : ce fut le cas pour l'énergie ou le temps. L'imaginaire était parfois sollicité dans le travail en guise de motivation de l'expérience ou d'application (réalisation d'un théâtre d'ombres pour le thème " ombre et lumière ").

Ainsi, pour la première séance en classe de $\mathrm{CP}$, nous avions demandé à chaque enfant d'apporter de chez lui des glaçons. 
>>

L'un d'eux est arrivé avec un sac plein d'eau et de surcroît troué, ce qui rendait la scène assez amusante ! D'autres s'étaient visiblement renseignés auprès de leurs parents et avaient apporté une véritable glacière!

«Pourquoi certains glaçons ont-ils fondu alors que d'autres sont restés presque intacts ?" avons-nous demandé à la classe. De là ont suivi de nombreuses expériences pour déterminer les facteurs intervenant dans ce phénomène surprenant !

La question suivante a été : "Comment faire pour faire fondre le glaçon plus ou moins vite ? " Les élèves ont alors suggéré d'enfermer le glaçon dans un pull, des sacs plastiques ou du papier aluminium (fig. 2), ou encore de le placer sous une lampe. Peu à peu, se dessinaient des interrogations autour de la notion de chaleur, qu'ils appréhendent en général plutôt à la manière d'un fluide !

Certaines années, nous avons même mis à profit les conditions climatiques en utilisant pour nos expériences la neige qui venait tout juste de tomber dans la cour de l'école ! Un autre jour, où brillait un grand soleil, nous sommes descendus dans la cour observer les ombres (thème " ombre et lumière ").

D'autres fois encore, nous sommes partis d'une observation : "Où va l'eau des flaques après la pluie ? D'où vient l'eau des rivières ? Que devient l'eau du linge quand il sèche? "Nous avons ensuite fait plusieurs expériences sur l'évaporation de l'eau en plaçant une même quantité d'eau dans des récipients de formes diverses, fermés ou pas, dans différent lieux (sur le radiateur, à l'extérieur, dans le réfrigérateur). Nous avons ainsi cherché à comprendre ce qui influençait le phénomène.

Mais quelle que fut la méthode employée, l'essentiel pour cette partie introductive du thème était, après avoir suscité un questionnement, d'amener ensuite les enfants à envisager eux-mêmes les moyens expérimentaux pour tester leurs hypothèses et tirer des conclusions.

\section{Deux exemples de séquences}

À titre d'exemples, nous allons maintenant décrire le déroulement de deux séquences types sur les thèmes du son (abordé en CE1 sur 5-6 séances environ) et de la mesure du temps et de l'espace (abordé en CP-CE1).

\section{Le son}

Nous débutons la première séance en posant à la classe des questions autour du son : Qu'est-ce que le son ? Pourquoi entend-on "des trucs»? Comment produit-on un son?

Suite à une première phase de réflexion autour du thème conduisant à de multiples pistes (l'écho, sons entendus par le bébé dans le ventre de sa mère...), nous demandons d'identifier les différentes façons de le produire. Les élèves ont pour cela le choix de (presque !) tout le matériel disponible dans la classe.

Nous laissons tout d'abord les élèves chercher différentes façons de produire un son, ce qui se traduit par une séquence vraiment très sonore ! Puis, au terme d'une mise en commun, nous élaborons ensemble un tableau pour identifier ce qui produit le son : les battements du cœur, le vent dans les arbres ou encore la peau d'un tambour...

Pour la séance suivante, la maîtresse demande à chacun d'apporter un objet qui produit un son. Certains élèves viennent avec un instrument de musique, d'autres avec des objets beaucoup plus incongrus !

Après une nouvelle phase d'expérimentation quelque peu bruyante, la discussion sur la façon de produire un son reprend. Nous réfléchissons ensemble, en particulier sur le fonctionnement des différents instruments de musique : guitare, xylophone, flûte et tambour. Cela nous permet ensuite de classer les différents sons dans un tableau, en fonction de l'action associée pour le produire :

Frapper, cogner - Souffler - Frotter Eau qui coule - Briser, casser.

À ce stade, plusieurs caractéristiques du son ont été proposées telles que : "Le son se déplace ", " se disperse ", " rebondit " (écho), ou encore que celui-ci a «quelque chose à voir avec l'air ".

Nous essayons alors de faire travailler les enfants différemment en explorant les possibilités sonores d'un même objet, ce que nous faisons en distribuant à chaque groupe des bouteilles vides en plastique. La consigne est très simple : trouver différentes façons de produire un son avec cet objet particulier. Ils s'en donnent à cœur joie, en tapant avec ou sur la bouteille, en soufflant dessus, en la remplissant d'eau ou en la frottant contre un autre objet.

Peu à peu, au fil de ces expériences un mécanisme est identifié et proposé par les élèves : la notion de "vibration ». Cette idée est approfondie de manière expérimentale avec des lames de xylophone en induisant une vibration que l'on peut sentir avec les doigts, et que l'on peut atténuer par une légère pression.

Pour clore ce thème de façon pédagogique, deux élèves ont préparé un exposé sur le fonctionnement de l'appareil auditif et des cordes vocales...

\section{Mesure du temps et de l'espace}

Nous débutons la séquence par une petite réflexion thématique : Qu'est-ce que le temps ? Quels phénomènes se répètent ?

Après cette première discussion, nous demandons aux élèves de dessiner leur vision personnelle du temps. Il s'ensuit un nouvel échange collectif sur la base des travaux de quelques-uns, qui viennent présenter leur dessin à tour de rôle à la classe.

Puis nous les questionnons à propos de la notion de mesure, thème que nous souhaitons aborder maintenant : C'est quoi mesurer quelque chose? Comment mesurer le temps?

Afin d'entrer véritablement dans le vif du sujet et dans l'expérimentation, nous posons un défi aux enfants :

"Imaginons que nous sommes des spéléologues et que nous aimerions mesurer le temps... Comment procéder si, par malheur, nous avions oublié notre montre à l'extérieur?"

Pour cela, on fournit le matériel suivant : de petites bouteilles en plastique, de la semoule... Les élèves s'attèlent alors à la fabrication d'un sablier. Nous passons ensuite un certain temps à comprendre ensemble ce qui ne fonctionne pas bien et à optimiser leurs réalisations.

À ce stade, nous proposons un nouveau défi : mesurer la durée d'une chanson ! En comparant les mesures des différents groupes d'élèves, on parvient à l'idée qu'il est nécessaire d'établir un étalon de mesure. Les élèves proposent d'eux-mêmes d'utiliser la durée d'une chanson très courte pour donner l'unité de temps. L'étalon de temps est appelé « le canard »!!! Après ce travail sur le temps, nous souhaitons aborder la notion d'espace et la mesure d'une longueur. Nous commençons par débattre du sens du mot " espace ", puis vient un exercice de représentation personnelle de cette notion. Ce petit travail nous conduit à une réflexion collective autour de la notion de " grand » et « petit »... Tout est relatif! 


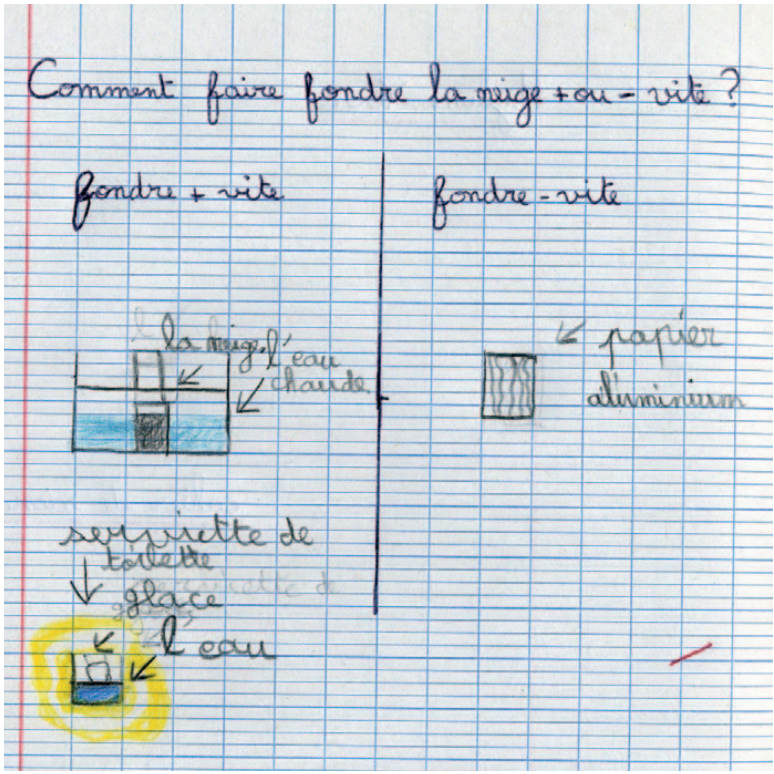

2. Schéma extrait du cahier d'expériences d'un élève, lors d'une séance sur les états de l'eau.

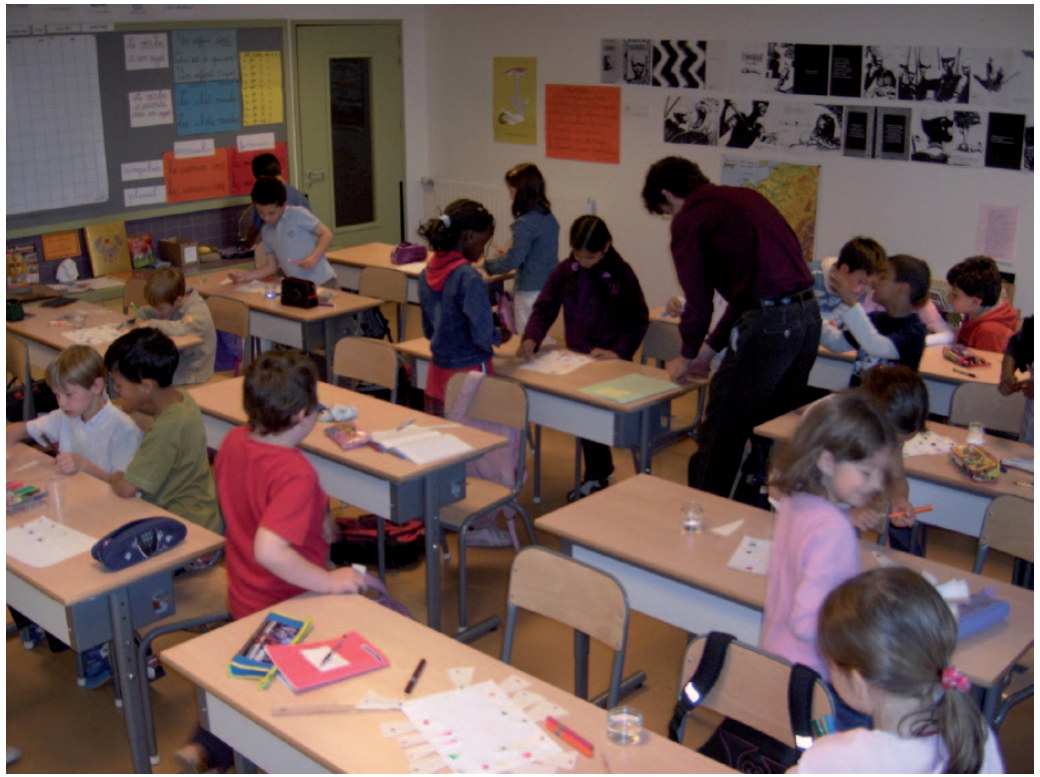

3. La classe en plein travail pendant un atelier.
Nous proposons ensuite un tout premier défi : Comment mesurer un objet sans règle ? Les idées fusent et les élèves utilisent les objets les plus saugrenus! Le deuxième défi consiste à mesurer la longueur de la table sans règle... Pas si facile!

Comme pour la mesure du temps, les élèves parviennent naturellement à la conclusion qu'un étalon de mesure est nécessaire afin de pouvoir comparer les résultats des différentes mesures de chaque groupe.

Dans un deuxième temps, nous demandons aux élèves de représenter leur table sur une feuille A3, puis de comparer leur dessin avec leur table réelle. On en vient ainsi à la notion de "plan ", dont nous expliquons l'utilité. La séquence se termine par un travail de groupe, qui consiste à représenter à l'échelle le plan de la classe sur une feuille A3.

Au terme de ce travail, il semble que les élèves aient bien compris les notions de mesure et d'étalon de mesure du temps ou de la longueur.

\section{Vers une école plus humaine et plus créative?}

Celui « qui n'est plus capable de s'émerveiller et de s'étonner, est en quelque sorte mort, et son œil est éteint " disait Einstein [2]. Dans le sillage de cet illustre savant, la curiosité et son développement au sein de l'école nous semblent être le cœur du processus éducatif. Or, l'éducation actuelle, malgré certaines évolutions ces quarante dernières années, est toujours principalement fondée sur une transmission passive du savoir, le développement de la créativité restant très peu présent.

Nous pensons que les ateliers science proposent une toute autre façon d'aborder la connaissance. Ils favorisent en effet l'ouverture sur le monde, la réflexion, l'imagination, l'esprit critique, le sens du collectif (notamment par le travail en groupe et les mises en commun). L'élève est actif : il réfléchit, invente, crée, regarde autour de lui, détourne les objets. Il se pose des questions et imagine par lui-même les moyens pour y répondre, dans une confrontation au réel et à l'autre qui se révèle toujours instructive.

D'autre part, la relation maitre-élève est très différente de celle d'un cours magistral. Par le biais des échanges individuels avec les élèves et du travail en petits groupes (fig. 3) se tissent naturellement des liens entre élèves et avec les enseignants, et ceci nous parait fondamental.

En effet, chaque enseignant a pu constater que nul ne peut apprendre s'il n'a pas la motivation nécessaire, autrement dit s'il n'est pas animé par l'envie de connaitre et de comprendre. En d'autres termes, le désir d'apprendre, de découvrir doit avoir un certain sens pour l'élève. Ce désir s'acquiert avant tout dans le cercle familial, à travers les relations parents-enfants autour de la lecture et du savoir. Cependant, il faut faire en sorte que l'école soit aussi un lieu où cette dimension puisse éclore et bourgeonner. Encore faut-il pour cela accepter que le côté " humain » ait pleinement sa place et ne soit pas négligé.

L'auteur autrichien Hugo von Hofmannsthal écrivait cette très belle phrase : "Toute rencontre me brise et me recompose. " Il en va de même pour chaque connaissance véritable que nous acquérons, qui nous conduit à reconstruire à chaque fois un édifice en intégrant cette nouvelle pierre. Cette intégration nous est rendue possible par l'exercice de notre faculté de penser. Mais encore faut-il apprendre à bien penser, et ce en relation étroite avec la réalité qui nous entoure. Cette rigueur, la démarche de la physique expérimentale l'exige en permanence, et elle devient un modèle transposable, non seulement dans de nombreux domaines du savoir, mais également dans la vie quotidienne.

Les ateliers science menés à l'école élémentaire Boucicaut ne visaient pas tant la transmission d'un savoir scientifique que celle de la joie de découvrir et l'exercice exigeant de la pensée.

\section{Références}

1-A. Einstein, Comment je vois le monde, Éditions Flammarion (1999).

2• G. von Boehm, Qui était Albert Einstein ? Éditions Assouline (2005). 